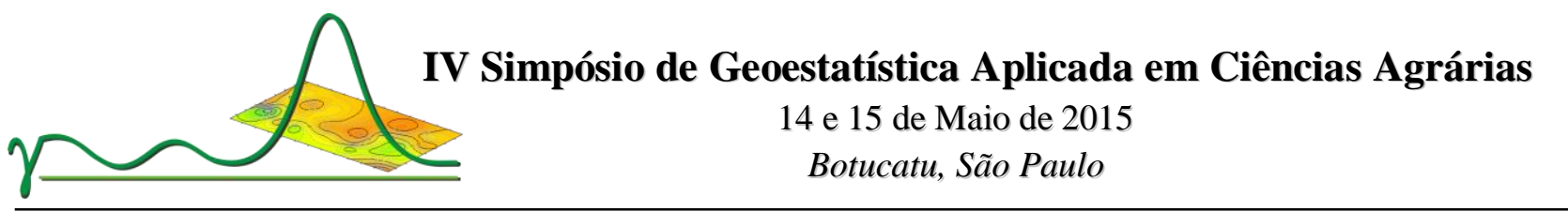

\title{
ANÁLISE ESPACIAL MULTIVARIADA APLICADA AO MAPEAMENTO DA PROBABILIDADE DE OCORRÊNCIA DO PERCEVEJO-DO-COLMO EM ARROZ IRRIGADO
}

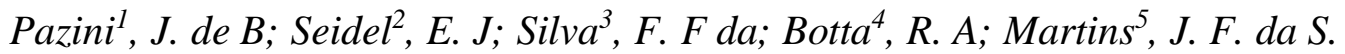

\begin{abstract}
${ }^{1}$ Engenheiro Agrônomo, Mestrando no Programa de Pós-Graduação em Fitossanidade, Universidade Federal de Pelotas, Departamento de Fitossanidade, Caixa Postal 354, CEP 96010-900, Pelotas, RS, Brasil, julianopazzini@hotmail.com

${ }^{2}$ Matemático, Professor Adjunto, Universidade Federal de Santa Maria, Departamento de Estatística, Cidade Universitária, CEP 97105-900, Santa Maria, RS, Brasil, enioseidel@gmail.com

${ }^{3}$ Engenheiro Agrônomo, Professor Associado, Universidade Federal do Pampa/Campus de Itaqui, fernandosilva@unipampa.edu.com.br

${ }^{4}$ Engenheiro Agrônomo, Mestrando no Programa de Pós-Graduação em Entomologia, Universidade Federal de Pelotas, Departamento de Microbiologia e Parasitologia, Caixa Postal 354, CEP 96010-900, Pelotas, RS, Brasil, robson_a.b@hotmail.comUniversidade Federal do Pampa/Campus de Itaqui, robson_a.b@hotmail.com

${ }^{5}$ Engenheiro Agrônomo, Doutor, Pesquisador, Embrapa Clima Temperado, Caixa postal 403, CEP 96001-970, Pelotas, RS, Brasil, jose.martins@embrapa.br

Resumo - O objetivo do presente trabalho foi determinar a probabilidade de ocorrência de Tibraca limbativentris Stal 1860 (Hemiptera: Pentatomidae) em arroz irrigado por inundação na região do Planalto da Campanha do Rio Grande do Sul, no contexto multivariado. O monitoramento do inseto ocorreu num talhão de lavoura de arroz de 13,7 ha, utilizando-se uma grade com 81 pontos georreferenciados e equidistantes $50 \mathrm{~m}$, nas fases vegetativa e reprodutiva da cultura e pós-colheita. O número de percevejos de cada ponto foi transformado, codificando os valores em zero (0) (ausência do inseto) e um (1) (presença do inseto). Primeiramente, foi determinada a probabilidade de ocorrência para as variáveis adultos, ninfas e adultos + ninfas, como produto das probabilidades de cada época de amostragem, por meio da regra multiplicativa de probabilidades para eventos independentes (épocas de amostrais). Após, realizou-se análise geoestatística via ajuste de semivariogramas e interpolação dos dados por krigagem ordinária. A probabilidade de ocorrência de adultos, ninfas e adultos + ninfas de T. limbativentris em arroz irrigado por inundação é maior nas zonas marginais da lavoura, no entanto, o detalhamento espacial da probabilidade de ocorrência do inseto foi prejudicado pelo método de avaliação espacial multivariada, com análise simultânea das épocas de amostragem.
\end{abstract}

Palavras-chave: geoestatística; amostragem; Oryza sativa.

\section{USE OF MULTIVARIATE SPATIAL ANALYSIS TO MAPPING THE PROBABILITY OF OCCURRENCE OF STEM BUG IN FLOODED RICE FIELD}

\begin{abstract}
The aim of this study was to determine the occurrence probability of Tibraca limbativentris Stal 1860 (Hemiptera: Pentatomidae) in flooded rice field in "Planalto da Campanha" Region, Rio Grande do Sul (RS), Brazil, in the multivariate context. The insect monitoring occurred in a 13,7 ha plot of rice crop, using a grid with 81 georeferenced points and equidistant $50 \mathrm{~m}$. The number of stem bugs of each points was transformed, encoding the values to zero (0) (insect absence) and (1) (insect presence). First, it was determined the occurrence probability for adults, nymphs and adults + nymphs variables as product of the probabilities of each sampling period, using the multiplicative probabilities rule for independent events (sampling periods). After, it was performed geostatistical analysis by semivariogram fit and data interpolation by ordinary kriging. The probability of occurrence of adults, nymphs and adults + nymphs of T. limbativentris in flooded rice is larger in the marginal zones of rice field, however, the spatial details of insect occurrence probability was harmed by multivariate spatial assessment method, with simultaneous analysis of sampling periods.
\end{abstract}

Key words: rice stem bug; sampling; Oryza sativa.

\section{Introdução}

Em sistemas de arroz irrigado por inundação no Estado do Rio Grande do Sul, o percevejo-do-colmo Tibraca limbativentris Stal 1860 (Hemiptera: Pentatomidae), também conhecido como percevejo-marrom, percevejo-grande-doarroz ou percevejo-das-hastes, está entre as espécies de insetos mais prejudiciais à cultura (MARTINS et al., 2004), onde para cada inseto adulto/m², em média, é esperada uma redução de 1,2\% na produtividade (REUNIÃO, 2014). Esta praga ocorre mais frequentemente entre V4 a R4, estádios correspondentes à época que vai do início do perfilhamento 


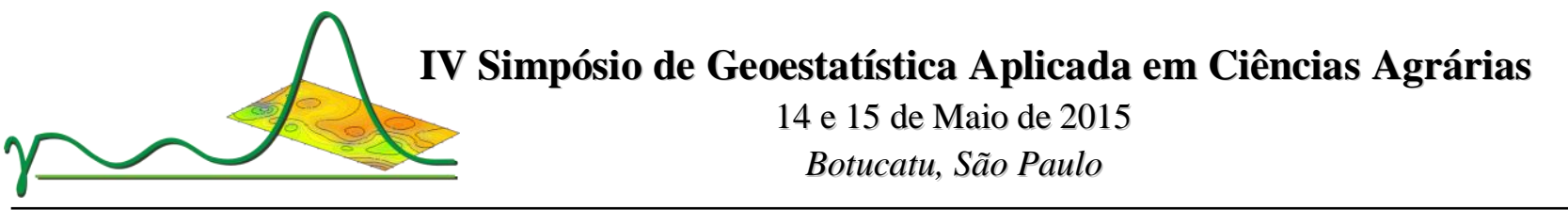

até a floração (COUNCE et al, 2000; REUNIÃO, 2014) e os danos causados são consequências de seu hábito alimentar, que já na fase ninfal ao alimentar-se das plantas introduz substâncias tóxicas, onde na fase vegetativa causa o sintoma chamado "coração-morto" e na fase reprodutiva o sintoma "panícula-branca".

O principal método de controle deste inseto é por meio do emprego de inseticidas químicos, porém, sem considerar os princípios do Manejo Integrado de Pragas (MIP), sendo que em muitos casos as aplicações são realizadas sem monitoramento prévio da população da praga e em épocas não indicadas para o efetivo controle (MARTINS et al., 2009), resultando, assim, no manejo inadequado da praga.

A metodologia de monitoramento para $T$. limbativentris atualmente recomendada consiste na escolha aleatória de pontos de amostragem na lavoura, com distinções na técnica empregada relacionada, apenas, quanto a fase da cultura, vegetativa ou reprodutiva (REUNIÃO, 2014). No entanto, um plano de amostragem para tomada de decisão em manejo de pragas deve considerar, também, o comportamento espacial do inseto no campo (BOEVE; WEISS, 1998) a fim de que orientações padronizadas e seguras de amostragem sejam estabelecidas (FERNANDES et al., 2003). Assim, há necessidade de aprimorar a metodologia de monitoramento existente para T. limbativentris a partir do entendimento do comportamento espacial do inseto, de modo que a população da praga seja avaliada com eficiência e praticidade e, que a técnica empregada tenha boa aceitação pelos orizicultores e extencionistas (ZEISS; KLUBERTANZ, 1993).

Estudo recente realizado por Pazini et al. (2013), com o mapeamento da probabilidade de ocorrência de adultos e ninfas de T. limbativentris em três distintas fases - vegetativa, reprodutiva e pós-colheita (resteva) - da cultura do arroz irrigado, demonstrou inovação no aperfeiçoamento das técnicas de amostragem para T. limbativentris, em que o monitoramento georreferenciado e métodos geoestatísticos constituem uma importante ferramenta para o entendimento do comportamento espaço-temporal do inseto na lavoura e, com isso, a possibilidade da elaboração de protocolos para o monitoramento da praga. Sob esta perspectiva e considerando a possibilidade de estabelecer o mapeamento de probabilidade de ocorrência do percevejo-do-colmo em todo o ciclo produtivo do arroz, de modo único e não segmentado a cada fase da cultura, como o proposto por Pazini et al. (2013), objetivou-se com este trabalho determinar a probabilidade de ocorrência de $T$. limbativentris em arroz irrigado por inundação na região do Planalto da Campanha do Rio Grande do Sul, no contexto multivariado.

\section{Material e Métodos}

O presente trabalho foi realizado na safra de 2009/10, em lavoura comercial de arroz instalada num Luvissolo, com declividade média de 4,8\%, na Fazenda Pitangueira, situada a 2909'56.52"S e 56²9'20.06"W, em Itaqui, RS. O clima predominante da região é "Cfa", subtropical, temperado quente, com chuvas bem distribuídas e estações bem definidas, segundo classificação de Köppen-Geiger. A cultura foi implantada em cultivo mínimo, com a cultivar IRGA 417, na primeira semana do mês de outubro, na densidade de 60 sementes $/ \mathrm{m}$ linear, num espaçamento de $0,17 \mathrm{~m}$ entre linhas. A adubação foi de $286 \mathrm{~kg} \mathrm{ha}^{-1}$ de 4-17-27 N-P-K na semeadura, $150 \mathrm{~kg} \mathrm{ha}^{-1}$ de 45-0-0 N-P-K aos 15 dias pós-emergência das plântulas, antes da inundação do arrozal, e $75 \mathrm{~kg} \mathrm{ha}^{-1}$ de 30-0-20 N-P-K na diferenciação do primórdio floral. O manejo fitossanitário foi empregado conforme as recomendações técnicas para a cultura do arroz irrigado (REUNIÃO, 2014), no entanto, sem aplicações de inseticidas. No manejo pós-colheita do arroz, em meados de fevereiro, a lavoura foi submetida a duas passadas de grade aradora e uma de grade niveladora para destruição dos restos culturais (resteva).

Para execução do monitoramento de T. limbativentris na lavoura de arroz, foi estabelecido, previamente, um grid mais ou menos regular com 81 pontos georreferenciados e equidistantes $50 \mathrm{~m}$, conforme orientação adaptada de Kuno (1991), num talhão de lavoura de 13,7 ha. Os levantamentos populacionais da praga abrangeram as fases vegetativa e reprodutiva da cultura, como também após a colheita, ocorrendo nos dias 06/01/2010, 02/02/2010 e 08/03/2010 quando as plantas encontravam-se em V11 (estádio de formação do colar na 11 a folha do colmo principal), R6 (expansão de um ou mais grãos em profundidade) (COUNCE et al., 2000) e após a colheita, na resteva. Em cada ponto de amostragem lançou-se um quadrado de metal medindo $0,5 \mathrm{~m} \mathrm{x} 0,5 \mathrm{~m}\left(0,25 \mathrm{~m}^{2}\right)$, onde as plantas inseridas na área do quadro foram examinadas visualmente a fim de realizar a contagem do inseto em sua fase jovem e adulta.

Primeiramente, os dados numéricos de adultos, ninfas e adultos + ninfas de cada ponto, foram transformados em indicadores de probabilidade, isto é, em níveis de corte baseados na afirmativa de que para cada inseto adulto/ $\mathrm{m}^{2}$, em média, é esperada uma redução de 1,2\% na produtividade (REUNIÃO, 2014). Como níveis de corte os valores foram codificados em zero (0) quando da ausência do inseto e, com o valor 1, nas ocasiões de presença, originando, assim, novo conjunto de dados, onde as contagens de insetos $(0,1,2,3, \ldots)$, foram transformadas em probabilidades $(0,1)$ (Tabela 1).

Tabela 1. Valores observados e transformados para o número de adultos, ninfas e adultos + ninfas do percevejo-docolmo, dos cinco primeiros pontos de amostragem do levantamento do dia 06/01/2010. Safra 2009/10. Itaqui-RS. 


\begin{tabular}{|c|c|c|c|c|c|c|c|c|}
\hline & & & & $\begin{array}{r}\text { destat } \\
14 \text { e } 1 \\
\text { Bot }\end{array}$ & $\begin{array}{l}\text { tica Ap. } \\
\text { de Maio } \\
a t u, \text { São } 1\end{array}$ & $\begin{array}{l}\text { cada er } \\
2015 \\
\text { ulo }\end{array}$ & iência & rárias \\
\hline \multirow{3}{*}{$\begin{array}{l}\text { Pontos } \\
\text { amostrais }\end{array}$} & \multicolumn{2}{|c|}{ Coordenadas UTM } & \multicolumn{6}{|c|}{ Número de insetos } \\
\hline & \multirow[b]{2}{*}{ Easting } & \multirow[b]{2}{*}{ Northing } & \multicolumn{3}{|c|}{ Observados } & \multicolumn{3}{|c|}{ Transformados } \\
\hline & & & Adultos & Ninfas & $\begin{array}{l}\text { Adultos } \\
+ \text { Ninfas } \\
\end{array}$ & $\begin{array}{c}\mathbf{P} \\
\text { (Adultos) }\end{array}$ & $\begin{array}{c}\mathbf{P} \\
\text { (Ninfas) }\end{array}$ & $\begin{array}{l}\text { P (Adultos } \\
+ \text { Ninfas) }\end{array}$ \\
\hline 1 & 549407,491 & 6773616,577 & 0 & 0 & 0 & 0 & 0 & 0 \\
\hline 2 & 549358,116 & 6773631,101 & 0 & 1 & 1 & 0 & 1 & 1 \\
\hline 3 & 549307,156 & 6773643,032 & 5 & 0 & 5 & 1 & 0 & 1 \\
\hline 4 & 549258,853 & 6773658,527 & 0 & 0 & 0 & 0 & 0 & 0 \\
\hline 5 & 549215,609 & 6773673,377 & 2 & 3 & 5 & 1 & 1 & 1 \\
\hline
\end{tabular}

De acordo com a abordagem apresentada por Landim e Sturaro (2002) sobre krigagem indicativa no contexto multivariado, primeiramente, foi determinada a probabilidade de ocorrência para cada variável (Adultos, Ninfas e Adultos + Ninfas), como produto das probabilidades de cada época de amostragem, por meio da regra multiplicativa de probabilidades para eventos independentes, como, no caso, as épocas de amostragem, usando as expressões a seguir:

$P($ Adultos $)=P($ Adultos 06/01/2010 $) * P($ Adultos 02/02/2010 $) * P($ Adultos 08/03/2010 $)$;

$P($ Ninfas $)=P($ Ninfas 06/01/2010 $) * P($ Ninfas 02/02/2010 $) * P($ Ninfas 08/03/2010 $)$;

$P($ Adultos + Ninfas $)=P($ Adultos + Ninfas 06/01/2010 $) * P($ Adultos + Ninfas $02 / 02 / 2010) * P($ Adultos + Ninfas 08/03/2010).

Os dados de adultos, ninfas e adultos + ninfas transformados foram submetidos à análise geoestatística por meio do pacote geoR (RIBEIRO JÚNIOR; DIGGLE, 2001) do software R (R DEVELOPMENT CORE TEAM, 2011), e utilizando o estimador robusto de semivariâncias (CRESSIE; HAWKINS, 1980) foram elaborados gráficos de semivariogramas experimentais para a avaliação da dependência espacial, considerando simultaneamente as três épocas de amostragem.

Após a obtenção dos semivariogramas, foram ajustados modelos matemáticos teóricos, sendo esses: esférico, exponencial e gaussiano (YAMAMOTO; LANDIM, 2013). Nos casos de independência espacial, ajustou-se ao modelo de efeito pepita puro.

A avaliação do melhor modelo teórico ajustado foi realizada visualmente, ou seja, "ajuste a sentimento" (ZIMBACK, 2001), sendo repetido até que fosse encontrado um modelo considerado satisfatório.

A qualidade dos ajustes foi determinada pelo índice de dependência espacial (IDE), proposto por Seidel e Oliveira (2014), o qual contempla uma medida de correlação espacial e considera todos os parâmetros dos modelos de semivariograma.

Com a definição e ajuste dos modelos utilizou-se a krigagem ordinária na interpolação dos dados transformados (equivalente a krigagem indicativa) para geração de mapas probabilísticos de ocorrência do percevejo-do-colmo (YAMAMOTO; LANDIM, 2013). Além disso, a variabilidade das predições foi avaliada por meio de mapas de variâncias de krigagem ordinária gerados.

\section{Resultados e Discussão}

Considerando as três épocas de amostragem simultaneamente, observou-se dependência espacial para as variáveis adultos, ninfas e adultos + ninfas, com ajuste do modelo matemático gaussiano aos semivariogramas dos dados (Tabela 2).

Tabela 2. Parâmetros estimados dos modelos ajustados aos semivariogramas experimentais para adultos, ninfas e adultos + ninfas de T. limbativentris, em arrozal irrigado por inundação, na safra 2009/10. Itaqui-RS.

\begin{tabular}{lccccccc}
\hline Inseto-praga & Modelo & $\begin{array}{r}\text { Efeito } \\
\text { pepita } \\
\left(\mathbf{C}_{\mathbf{0}}\right)\end{array}$ & $\begin{array}{c}\text { Patamar } \\
\left(\mathbf{C}_{\mathbf{0}}+\mathbf{C}_{\mathbf{1}}\right)\end{array}$ & Alcance (a) & $\begin{array}{c}\text { Máxima } \\
\text { Distância }\end{array}$ & Hmax $^{\mathbf{2}}$ & IDE (\%) $^{\mathbf{1}}$ \\
\hline Adultos & Gaussiano & 0,0000 & $1,2 \times 10^{-6}$ & $321,51 \mathrm{~m}$ & $575,55 \mathrm{~m}$ & $\begin{array}{c}0,70 * 575,55= \\
402,89 \mathrm{~m}\end{array}$ & 50,4 \\
Ninfas & Gaussiano & 0,0000 & $2,1 \times 10^{-5}$ & $498,05 \mathrm{~m}$ & $575,55 \mathrm{~m}$ & $\begin{array}{c}0,65 * 575,55= \\
374,11 \mathrm{~m}\end{array}$ & 50,4 \\
Adultos + Ninfas & Gaussiano & 0,0007 & 0,0057 & $650,55 \mathrm{~m}$ & $575,55 \mathrm{~m}$ & $\begin{array}{c}0,85 * 575,55= \\
489,22 \mathrm{~m}\end{array}$ & 44,2 \\
\hline
\end{tabular}

${ }^{1} \operatorname{IDE}(\%)=\mathrm{FM}^{*}\left(\mathrm{C}_{1} / \mathrm{C}_{0}+\mathrm{C}_{1}\right) *[\mathrm{a} /(0,5 * \mathrm{MD})]^{* 100} ; \mathrm{FM}=0,504$ no modelo gaussiano, em que FM é o fator do modelo, $\mathrm{MD}$ é a máxima distância entre pontos amostrados, $\mathrm{C}_{0}$ é o efeito pepita, $\mathrm{C}_{1}$ é a contribuição e a é o alcance prático. Observação: como o alcance foi maior que a metade da maior distância $\left(0,5^{*} \mathrm{MD}\right)$, nos três estádios de vida do inseto, o valor de $\mathrm{a} /(0,5 * \mathrm{MD})$ foi truncado em $1 .{ }^{2}$ Distância considerada no semivariograma estimado.

O alcance (a), que é a distância máxima da dependência espacial, e indica que a partir dessa distância termina a dependência entre os casos estudados foi de $321,51 \mathrm{~m}, 498,05 \mathrm{~m}$ e $650,55 \mathrm{~m}$ para adultos, ninfas e adultos + ninfas, 


\section{Simpósio de Geoestatística Aplicada em Ciências Agrárias \\ 14 e 15 de Maio de 2015 \\ Botucatu, São Paulo}

respectivamente, sendo estes valores próximos ou superiores a máxima distância no interior do grid de amostragem (Tabela 2), indicando alta correlação entre os pontos. As distâncias entre os pontos num procedimento de amostragem devem ser menores que os valores do alcance encontrados (VALERIANO; PRADO, 2001), pois representam a distância limite da dependência espacial (FERRAZ et al., 2012).

O critério de qualidade dos ajustes dos modelos matemáticos de semivariogramas proposto por Seidel e Oliveira (2014) indicou "alta" dependência espacial $\left(0 \leq \operatorname{IDE}_{\text {Gaussiano }}(\%) \leq 50,4\right)$, possibilitando, assim, a obtenção de mapas mais precisos para determinação da probabilidade de ocorrência do percevejo na lavoura (ASSIS, 2005).

A Figura 1 apresenta os semivariogramas indicadores e respectivos mapas de probabilidade de ocorrência de adultos, ninfas e adultos + ninfas de $T$. limbativentris gerados no contexto mutivariado, com a análise simultânea das três épocas de amostragem para cada variável.

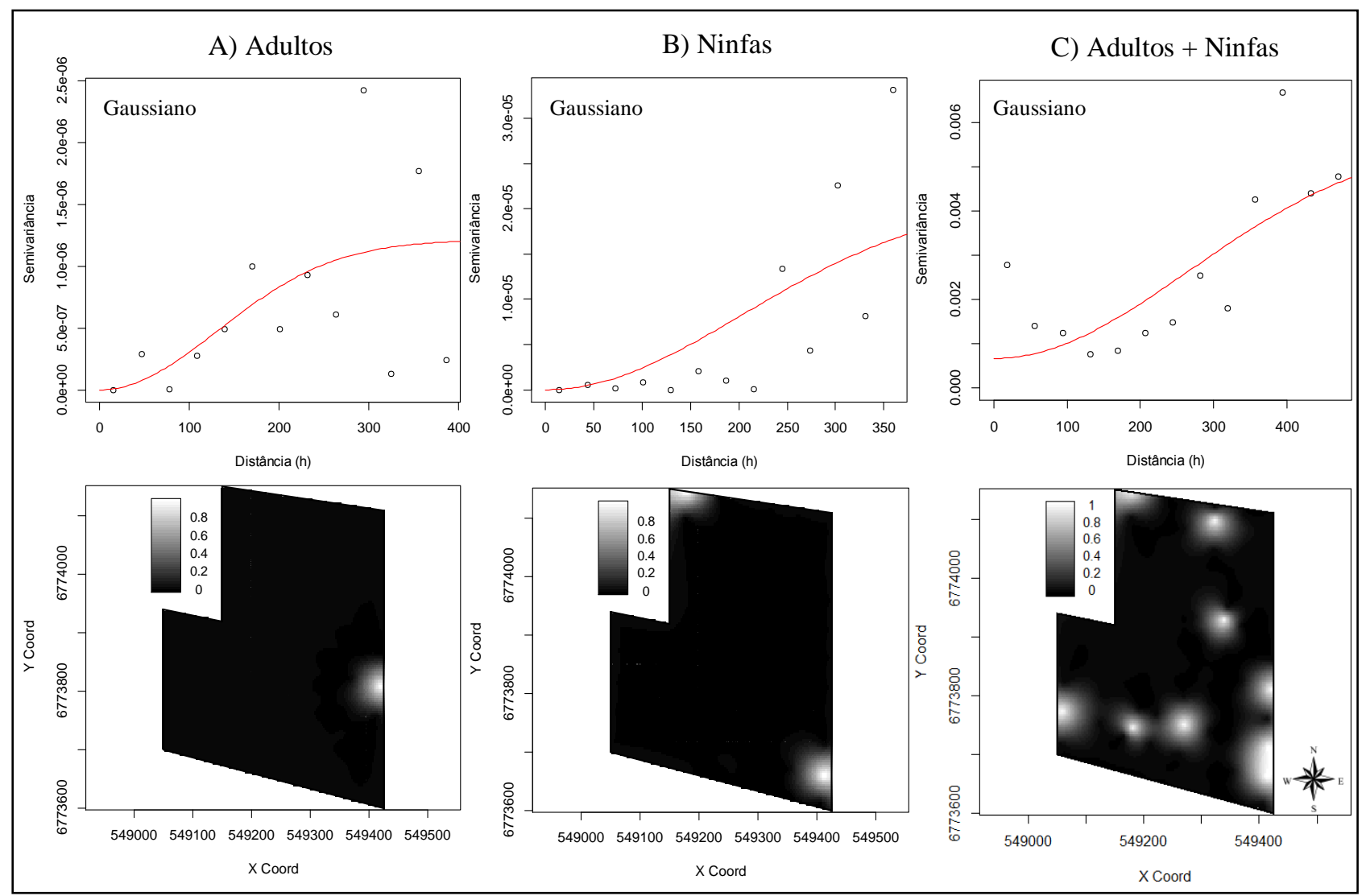

Figura 1. Semivariogramas indicadores e respectivos mapas de probabilidade de ocorrência de (A) Adultos, (B) Ninfas e (C) Adultos + Ninfas de T. limbativentris, em arrozal irrigado por inundação, na safra 2009/10. Itaqui, RS.

Observa-se, para adultos, ninfas e adultos + ninfas, a presença da praga, predominantemente, em focos nas regiões marginais (bordadura) da lavoura. Isso pode ser explicado em razão da proximidade destas zonas com o sítio de hibernação dos insetos, que localiza-se, principalmente, na vegetação espontânea no entorno da área. Ao migrarem, os adultos identificam estes locais no interior da lavoura como adequados para alimentarem-se ativamente e intensificarem a atividade sexual (FERREIRA, 1997). Como consequência disto, surgiram as ninfas, que por apresentarem incapacidade de voo e comportamento agregado, apresentaram comportamento espacial semelhante ao dos adultos, com focos de ocorrência também nas proximidades da bordadura da lavoura.

Comparativamente ao mapeamento realizado por Pazini et al. (2013), em que a probabilidade de ocorrência do inseto foi realizado por fase da cultura, nota-se que, embora a probabilidade de ocorrência do inseto seja maior na região marginal da lavoura, houve elevado prejuízo no detalhamento espacial da ocorrência de T. limbativentris na área ao analisar simultaneamente as épocas de amostragem. Isto porque, os dados populacionais coletados em pós-colheita apresentaram, em sua grande maioria, muitos pontos com nenhum inseto contabilizado, em razão dos efeitos mecânicos da colheita condicionar alta mortalidade da população da praga presente na lavoura (PAZINI et al., 2012). Com isso, a inserção desses dados numéricos da população do percevejo em pós-colheita para análise em conjunto com as demais épocas, resultou na diminuição da probabilidade de ocorrência da praga nas variáveis adultos, ninfas e adultos + ninfas.

Com relação as estimativas de probabilidade de ocorrência de adultos, ninfas e adultos + ninfas, detalhada nos mapas de variâncias de krigagem ordinária (Figura 2), onde as zonas mais escuras nos mapas representam menores 


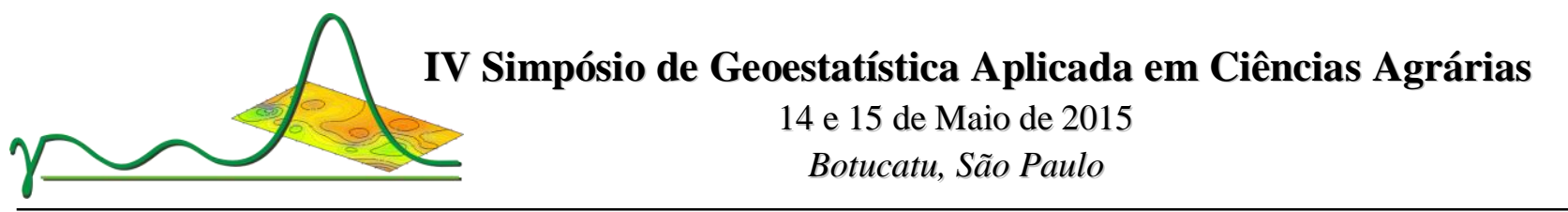

valores de variabilidade, isto é, menor erro na estimativa, apresentaram menores erros nas áreas do mapa com maior densidade de pontos amostrados, o que é esperado em monitoramentos populacionais.

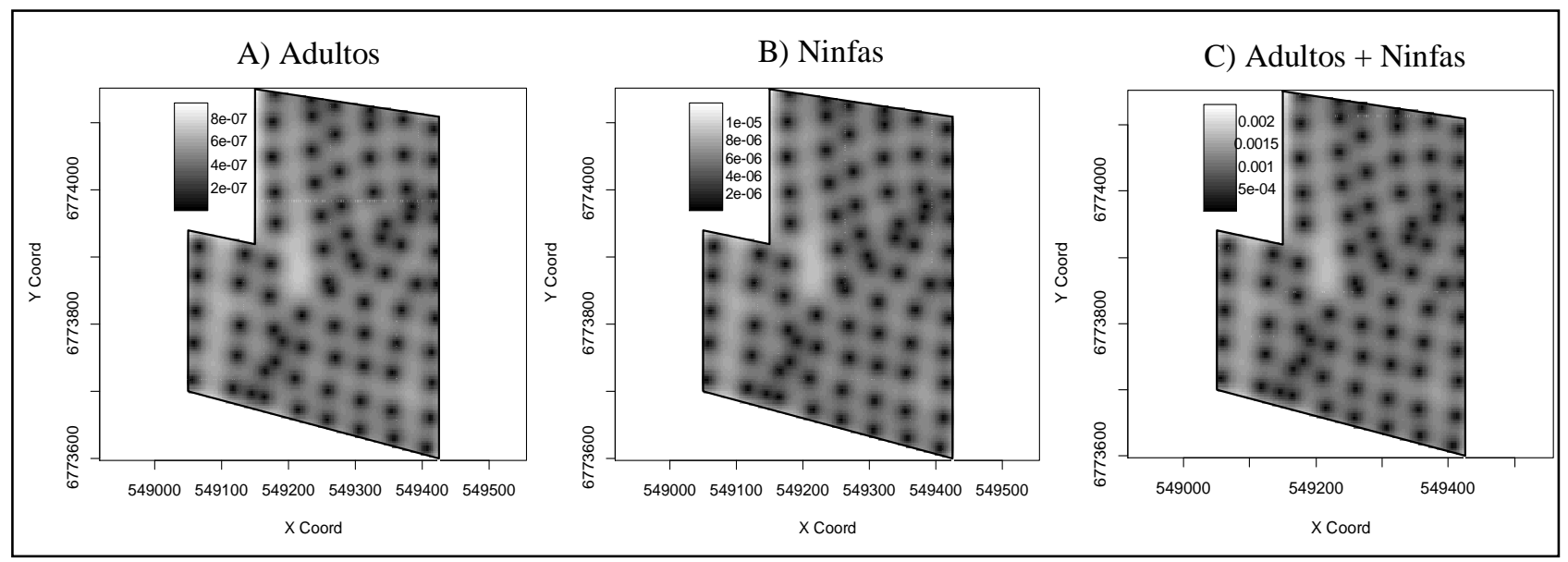

Figura 2. Mapas de variâncias de krigagem ordinária de (A) Adultos, (B) Ninfas e (C) Adultos + Ninfas de $T$. limbativentris, em arrozal irrigado por inundação, na safra 2009/10. Itaqui, RS.

\section{Conclusão}

A probabilidade de ocorrência de adultos, ninfas e adultos + ninfas de $T$. limbativentris em arroz irrigado por inundação é maior nas zonas marginais da lavoura.

$\mathrm{O}$ detalhamento espacial da probabilidade de ocorrência de adultos, ninfas e adultos + ninfas de T. limbativentris em arroz irrigado por inundação foi prejudicado pelo método de avaliação espacial multivariada, com análise simultânea das épocas de amostragem.

Novos estudos ainda são necessários a fim de comprovar a eficiência da abordagem multivariada para épocas de monitoramento em estudos de probabilidade de ocorrência de insetos-praga.

\section{Referências}

ASSIS, A. L. de. Definição da estratégia amostral em plantios jovens de Eucalyptus spp pelo uso de geoestatística. 2005. 118 f. Tese (Doutorado) - Curso de Doutorado em Engenharia Florestal, Universidade Federal de Lavras, Lavras, 2005. Disponível

em: <http://repositorio.ufla.br/bitstream/1/4051/1/TESE_Defini\%C3\%A7\%C3\%A3o\%20da\%20estrat\%C3\%A9gia\%20amo stral $\% 20 \mathrm{em} \% 20$ plantios $\% 20 \mathrm{de} \% 20$ Eucaliptus $\% 20 \mathrm{spp} \% 20$ pelo\%20uso\%20de\%20geoestat\%C3\%ADstica.pdf $>$. Acesso em: 05 dez. 2014.

BOEVE, P. J.; WEISS, M. Spatial distribution and sampling plans with fixed levels of precision for cereal aphids (Homoptera: Aphididae) infesting spring wheat. The Canadian Entomologist, v.130, n.1, p.66-77, 1998.

COUNCE, P.A.; KEISLING, T.C.; MITCHELL, A.J. A uniform, objective, and adaptative system for expressing rice development. Crop Science, v.40, p.436-443, 2000. Disponível em: 〈http://cses.uark.edu/ricepaper.pdf>. Acesso em: 07 dez. 2014.

CRESSIE, N.A.; HAWKINS, D.M. Robust estimation of the variogram: I. Mathematical Geology, v.12, n.2, p.115125, 1980. Disponível em: 〈http://www.ime.usp.br/ abe/lista/pdfOZDqFaTuKv.pdf〉. Acesso em: 12 dez. 2014.

FERNANDES, M.G.; BUSOLI, A.C.; BARBOSA, J.C. Distribuição espacial de Alabama argillacea (Hübner) (Lepidoptera:Noctuidae) em algodoeiro. Neotropical Entomology, v.32, n.1, p.107-115, 2003. Disponível em: 〈http://www.scielo.br/scielo.php?pid=S1519-566X2003000100016\&script=sci_arttext>. Acesso em: 15 dez. 2014. FERRAZ, G. A. S; SILVA, F. M. da; COSTA, P. A. N. da; SILVA, A. C.; CARVALHO, F. M. Agricultura de precisão no estudo de atributos químicos do solo e da produtividade de lavoura cafeeira. Coffee Science, v.7, n.1, p.59-67, 2012. Disponível em: 〈http://www.coffeescience.ufla.br/index.php/Coffeescience/article/view/204/pdf〉. Acesso em: $12 \mathrm{dez}$. 2014.

FERREIRA, E.; ZIMMERMANN, F.J.P.; SANTOS, A.B. dos; NEVES, B.P. das. O percevejo-do-colmo na cultura do arroz. Goiânia, Embrapa Arroz e Feijão, 1997. 43 p. (EMBRAPA-CNPAF. Documentos, 75). Disponível em: <http://ainfo.cnptia.embrapa.br/digital/bitstream/CNPAF/13155/1/doc_75.pdf>. Acesso em: 11 nov. 2014.

KUNO, E. Sampling and analysis of insect populations. Annual Review of Entomology, v.36, p.285-304, 1991. Disponível em: 〈http://www.annualreviews.org/doi/pdf/10.1146/annurev.en.36.010191.001441>. Acesso em: 07 ago. 2014. 


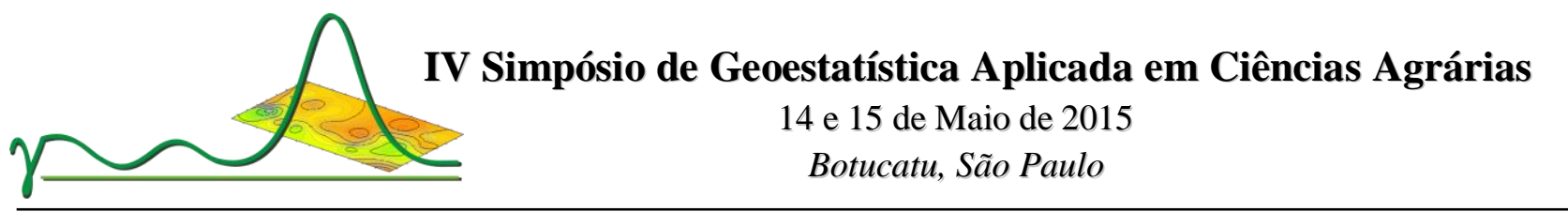

LANDIM, P.M.B.; STURARO, J.R. Krigagem indicativa aplicada à elaboração de mapas probabilísticos de riscos. DGA,IGCE,UNESP/Rio Claro, Lab. Geomatemática, Texto Didático 06, 19 pp. 2002. Disponível em <http://www.rc.unesp.br/igce/aplicada/textodi.html>. Acesso em: 15 nov. 2014.

MARTINS, J. F. da S.; BARRIGOSSI, J.A.F.; OLIVEIRA, J.V. de; CUNHA, U.S. da; Situação do manejo integrado de insetos-praga na cultura do arroz no Brasil. Pelotas: Embrapa Clima Temperado, 2009. 40p. (Embrapa Clima Temperado. Documentos, 290). Disponível em: <http://www.infoteca.cnptia.embrapa.br/bitstream/doc/783492/1/documento290.pdf>. Acesso em: 15 dez. 2014.

MARTINS, J.F. da S.; BOTTON, M.; CARBONARI, J.J.; QUINTELA, E.D. Eficiência de Metarhizium anisopliae no controle do Percevejo-do-colmo Tibraca limbativentris (Heteroptera: Pentatomidae) em lavoura de arroz irrigado. Ciência Rural, Santa Maria, v. 34, n. 6, p. 1681-1688, 2004. Disponível em: <http://www.scielo.br/scielo.php?script=sci_arttext\&pid=S1519-566X2008000100004>. Acesso em: 10 dez. 2014.

PAZINI, J. de B.; BOTTA, R. A.; SILVA, F. F. da. Mortalidade de percevejo-do-colmo do arroz no preparo do solo para cultivo mínimo. Pesquisa agropecuária brasileira, v.47, n.7, p.1022-1024, 2012. Disponível em: 〈http://www.scielo.br/pdf/pab/v47n7/47n07a20.pdf>. Acesso em: 23 nov. 2013.

PAZINI, J. de B.; SEIDEL, E.J.; BOTTA, R.A.; SILVA, F.F. da; MARTINS, J.F. da S. Mapeamento da probabilidade de ocorrência de Tibraca limbativentris em arroz irrigado por inundação. In: SIMPÓSIO DE GEOESTATÍSTICA APLICADA EM CIÊNCIAS AGRÁRIAS, 3., 2013, Botucatú. Anais... São Paulo: Universidade Estadual Paulista, Faculdade de Ciências Agronômicas, 2013. Disponível em: 〈http://www.fca.unesp.br/sgea/Docs2013/37.pdf>. Acesso em 20 dez. 2014.

R DEVELOPMENT CORE TEAM. $\quad \mathbf{R}$ - A language and environment for statistical computing. rev. 2.15.0. Vienna, Austria: R Foundation for Statistical Computing. 2011. Disponível em: 〈http://r-project.org>. Acesso em: 10 jan. 2013.

REUNIÃO TÉCNICA DA CULTURA DO ARROZ IRRIGADO, 30., 2014, Bento Gonçalves. Arroz irrigado: recomendações técnicas da pesquisa para o Sul do Brasil. Santa Maria: SOSBAI, 2014. 192p.

RIBEIRO JÚNIOR, P.J.; DIGGLE, P.J. geoR: a package for geostatistical analysis. R NEWS, v.1, n.2, p.15-18, 2001. Disponível em: 〈http://geodacenter.asu.edu/system/files/rnews1.2.15-18 0.pdf>. Acesso em: 11 jan. 2013.

SEIDEL, E.J.; OLIVEIRA, M.S. Novo índice geoestatístico para a mensuração da dependência espacial. Revista Brasileira de Ciência do Solo, v.38, n.3, p.699-705, 2014 . Disponível em: <http://www.scielo.br/scielo.php?script=sci_arttext\&pid=S0100-06832014000300002\&lng=pt\&nrm=iso >. Acesso em: 20 nov. 2014.

VALERIANO, M. M.; PRADO, H. Técnicas de geoprocessamento e de amostragem para o mapeamento de atributos anisotrópicos do solo. Revista Brasileira de Ciência do Solo, v.25, n.3, p.997-1005, 2001. Disponível em: 〈http://www.scielo.br/pdf/rbcs/v25n4/22.pdf >. Acesso em: 20 dez. 2014.

YAMAMOTO, J.K.; LANDIM, P.M.B. Geoestatística: conceitos e aplicações. São Paulo: Oficina de textos, 2013. ZEISS, M.R.; KLUBERTANZ, T.H. Sampling programs for soybean arthropods. In: PEDIGO, L.P.; BUNTIN, G.D. Handbook of sampling methods for arthropods in agriculture. Boca Raton: CRC, 1993. Cap.19. p.539-601.

ZIMBACK, C.R.L. Análise espacial de atributos químicos de solos para fins de mapeamento da fertilidade do solo. 2001. 114f. Tese (Livre-Docência em Levantamento do solo e Fotopedologia) - Universidade Estadual Paulista, Botucatu, SP. 